\title{
STUDY ON THE TWO-STAGE EXPANSION AIR-POWERED ENGINE
}

\author{
Hao LIU, Guoliang TAO and Ying Chen \\ The State Key Lab of Fluid Power Transmission and Control, Faculty of Engineering \\ Zhejiang University \\ 38 Zheda Road, Hangzhou ,310027, China \\ (E-mail: hliu2000@zju.edu.cn)
}

\begin{abstract}
Air-powered engine is a promising one of the low emission engines, for the advantages of its simple structure and complete zero-pollution. In this paper, the two-stage expansion Air-powered engine (TSEAPE) was studied in theory firstly and then experiments. Based on the model of TSEAPE that has been developed on the thermodynamics and mechanics analysis, the effects of bores ratio on the performance of TSEAPE are investigated by simulation. The results of simulation indicate that the TSEAPE will performance well when its bore ratio is about 1.4, which is a good balance between the power output and the efficiency. Guided by the results of theory analysis, a TSEAPE was developed based on an existing internal-combustion engine. The experiment results of this prototype engine on a test bench show that TSEAPE runs stably on bore ratio 1.4 and has better performance at a low speed.
\end{abstract}

\section{KEY WORDS}

Two-stage expiation air powered engine (TSEAPE), Low emission, Bores ration, Heat transfer

\section{NOMENCLATURE}

\section{$P_{i} \quad: \quad$ Injection air pressure}

$T_{i} \quad$ : Injection air temperature

$H_{i} \quad: \quad$ Injection air specific enthalpy

$M_{i}:$ Injection air mass

$P_{1}$ : The air pressure in the $1^{\text {st }}$ stage cylinder

$T_{1}$ : The air temperature in the $1^{\text {st }}$ stage cylinder

$m_{1}$ : The air mass in the $1^{\text {st }}$ stage cylinder

$V_{1}:$ The air volume in the $1^{\text {st }}$ stage cylinder

$u_{1}$ : Specific internal energy of the air in the $1^{\text {st }}$ stage cylinder

$h_{1}:$ Specific enthalpy of the air in the $1^{\text {st }}$ stage cylinder

$m_{\mathrm{e} 1}$ : $\quad$ Mass of the air out of the $1^{\text {st }}$ stage cylinder

$Q_{1}$ : Heat transfer of the $1^{\text {st }}$ stage cylinder

$W_{1}$ : Work of the $1^{\text {st }}$ stage cylinder

$\phi: \quad$ : Crank angle

$V_{\mathrm{h}}:$ Volume of the air in the heat-exchanger

$Q h$ : Heat transfer of the heat-exchanger $(\mathrm{J})$
$P_{2}:$ The air pressure in the $2^{\text {nd }}$ stage cylinder

$T_{2}$ : The air temperature in the $2^{\text {nd }}$ stage cylinder

$M_{2}$ : The air mass in the $2^{\text {nd }}$ stage cylinder

$V_{2}: \quad$ Volume of the air in the $2^{\text {nd }}$ stage cylinder

$u_{2}$ : Specific internal energy of the air in the $2^{\text {nd }}$ stage cylinder

$h_{2}: \quad$ Specific enthalpy of the exhaust air of the $2^{\text {nd }}$ stage cylinder

$m e_{2}: \quad$ Mass of the exhaust air out of the $2^{\text {nd }}$ stage cylinder

$Q_{2}: \quad$ Heat transfer of the $2^{\text {nd }}$ stage cylinder

$W_{2}$ : Work of the $2^{\text {nd }}$ stage cylinder

$\omega$ : Angular velocity of the crankshaft

$U$ : Gas internal energy

$\mathrm{R}$ : The gas constant for air

$\Omega \quad$ : $\quad$ Engine speed

$A_{w 1}$ : The total surface area of the first cylinder

$\mu$ : The coefficient of discharge

$A \quad$ : The opening area of the valve

$P_{\text {I }}: \quad$ The upstream pressure of the valve 
$\rho_{\mathrm{I}}: \quad$ The density of gas on the upstream

$\psi \quad$ : The mass flow coefficient

$P_{\text {II }} \quad: \quad$ The downstream pressure

$\mathrm{k} \quad: \quad$ The specific heat ratio

$a_{w}: \quad$ The coefficient of heat transfer applies to all surfaces of the cylinder

$T_{w}: \quad$ The surface temperature of the cylinders

$A_{w 2}: \quad$ The total surface area of the $2^{\text {nd }}$ cylinder

$a_{w h}: \quad$ The coefficient of heat transfer applies to the surface of the heat exchanger

$A_{w h}: \quad$ The total surface area of the heat exchanger

$P_{0}:$ : Barometric pressure

$S_{1} \quad$ : The projected area of the first piston

$\beta_{1}$ : The angle between the rod and the axis of the first cylinder

$\mu \quad$ : The coefficient of the sliding friction between piston and the wall of cylinder

$A_{b 1}$ : The contact area of the piston and the wall of the $1^{\text {st }}$ cylinder

$m_{i 1}$ : The reciprocating mass of the $1^{\text {st }}$ cylinder

$r:$ The radius of the crank

$\lambda$ : The ratio of crank radius to connecting rod.

$S_{2}$ : The projected area of the $2^{\text {nd }}$ piston

$\beta_{2}$ : The angle between the rod and the axis of the $2^{\text {nd }}$ cylinder

$A_{b 2}$ : The contact area of the piston and the wall of the $2^{\text {nd }}$ cylinder

$m_{i 2}$ : The reciprocating mass of the $2^{\text {nd }}$ cylinder

$E_{i}:$ The exergy of the high-pressure air injected into the cylinder.

\section{INTRODUCTION}

Air-powered engine (APE) uses high-pressure air as energy to operate. Therefore it is of completely zero emission and is becoming a promising candidate for low-emission vehicle propulsion [1].

Generally, two approaches are used to obtain a high efficient APE. One is to sufficiently utilize the energy of the high-pressure air. This goal is achieved by raising the expansion ratio of the air in a single working cycle, which reduces the energy loss with lower air pressure exhaust. The other approach is to obtain extra energy from the ambient. As the air temperature falls greatly in the expansion process which is not isothermal in the cycle, heat can be absorbed from the ambient to enhance the power output. This approach depends mostly on the expansion time and the heat transfer area, but these contributing factors are limited in the single-stage expansion APE. However, the two-stage expansion APE (TSEAPE) integrates the two approaches by expanding the inlet high-pressure air twice in two cascaded cylinders to raise the total expansion ratio which prolongs the time of the air stay in engine and enlarges the total heat transfer area.
With an increasing demand of low-emission vehicles, APE is being studied by many researchers all over the world in recent years and a lot of achievements have been obtained [2-4]. However, most of them are about the single-stage expansion APE except the MDI Company, a French company which has studied Air-powered Vehicle study for over 10 years and has declared a scheme of three-stage expansion APE, but no more details can be found [5]. Yet a lot of factors need to be considered in designing the engine. In this paper, it is of primary importance to make clear the effect of the ratio of expansion volumes on the performance of two-stage expansion APE. As to the reciprocating APE, which is the most common structure because of its reliable structure and nice air tightness, the ratio of expansion volumes is represented by the bores ratio of the cascaded cylinders in deferent stages. Therefore, the effect of bores ratio on the basic multi-stage expansion APE, two-stage expansion APE (TSEAPE), was studied with simulation method, based on the model developed from thermodynamic analysis of the engine. And a prototype TSEAPE was manufactured and experiments were carried out to verify the validity of the model in this paper.

\section{THE MODEL OF TSEAPE}

The scheme of reciprocating TSEAPE shows as Figure1. In each cylinder, the operating cycle is finished in two strokes, the intake stroke and exhaust stroke. The deference of pistons' moving phases in the two cylinders is $180^{\circ}$ which ensures the two-stage expansion.

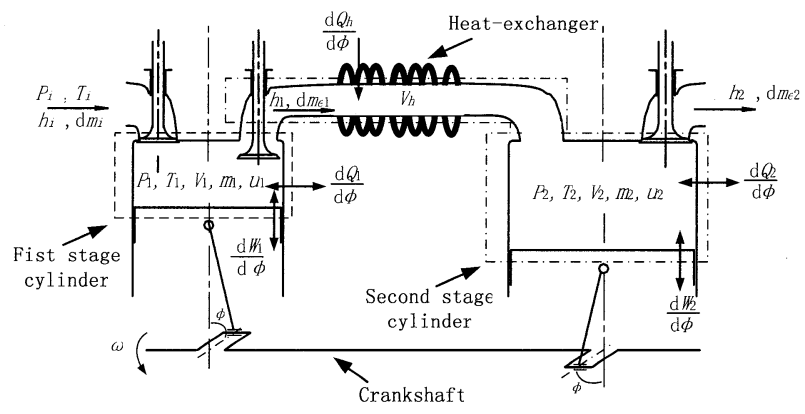

Figure 1 Scheme of TSEAPE

The operating cycle of TSEAPE is a thermodynamic process so that the model of TSEAPE is built on it in this paper. As high-pressure air expands twice in the two cylinders, the model of TSEAPE is composed of two closely linked sub-models. The two sub-models describe the operating processes in the two expansion spaces which are defined as control volumes and marked out with dashed lines. The first control volume is bounded 
by the piston and walls of the first stage cylinder. Because both the inner spaces of heat-exchanger and second stage cylinder are connected directly by the wide inlet port, the air in the two spaces is considered to be the same state in this paper.

\section{Sub-model of the first expansion stage}

The instantaneous state of the high-pressure air in the control volume is determined by energy conservation,

$$
\mathrm{d} U_{1}=\mathrm{d} Q_{1}+\mathrm{d} W_{1}+h_{i} \mathrm{~d} m_{i}+h_{1} \mathrm{~d} m_{1}
$$

The gas internal energy changes accordingly with its instantaneous mass and specific energy, the relation of them is expressed as:

$$
\mathrm{d} U_{1}=\mathrm{d}\left(m_{1} \cdot u_{1}\right)=u_{1} \mathrm{~d} m_{1}+m_{1} \mathrm{~d} u_{1}
$$

As we know, the gas specific energy $u$ is the function of pressure, temperature and component. However, the gas in the control volume is pure air and no component changes in the process, the function can be simplified as:

$$
u_{1}=u\left(T_{1}, P_{1}\right)
$$

Differential Eq. (3) can be expressed as:

$$
\mathrm{d} u_{1}=\left(\frac{\partial u_{1}}{\partial T_{1}}\right)_{P_{1}} \mathrm{~d} T_{1}+\left(\frac{\partial u_{1}}{\partial P_{1}}\right)_{T_{1}} \mathrm{~d} P_{1}
$$

Because the influence of pressure $P$ applying on the specific energy $u$ is very little, the change of the specific energy $u$ with the pressure can be neglected in our analysis. So when we insert Eq. (4) into Eq. (2), then insert rewritten Eq. (2) into Eq. (1), and for the convenience of analysis in a single cycle, differentiate the equation we obtained to the crank angle $\Phi$ and rearrange it, the following differential equation can be obtained as:

$$
\frac{\mathrm{d} T_{1}}{\mathrm{~d} \phi}=\frac{1}{m\left(\frac{\partial u_{1}}{\partial T_{1}}\right)}\left[\frac{\mathrm{d} Q_{1}}{\mathrm{~d} \phi}+\frac{\mathrm{d} W_{1}}{\mathrm{~d} \phi}+h_{i} \frac{\mathrm{d} m_{i}}{\mathrm{~d} \phi}+h_{1} \frac{\mathrm{d} m_{e 1}}{\mathrm{~d} \phi}-u_{1} \frac{\mathrm{d} m_{1}}{\mathrm{~d} \phi}\right]
$$

And considering the specific energy $u$ has the relation with the constant-volume specific heat $C_{v}$ as follows,

$$
\frac{\partial u}{\partial T}=C_{v}
$$

Then Eq. (5) can be rewritten as:

$$
\frac{\mathrm{d} T_{1}}{\mathrm{~d} \phi}=\frac{1}{m C_{v}}\left[\frac{\mathrm{d} Q_{1}}{\mathrm{~d} \phi}+\frac{\mathrm{d} W_{1}}{\mathrm{~d} \phi}+h_{i} \frac{\mathrm{d} m_{i}}{\mathrm{~d} \phi}+h_{1} \frac{\mathrm{d} m_{e 1}}{\mathrm{~d} \phi}-u_{1} \frac{\mathrm{d} m_{1}}{\mathrm{~d} \phi}\right]
$$

With the differential equation, the relationship between the gas state variable $T$ and the procedure variables is very clear. The items in the right of the equation can be defined in functions that have the crank angle $\Phi$ as variable. They are presented as follows.
The heat obtained by the gas in the control volume is mainly the result of the heat transferring between the cylinder surface and the gas. It is determined as:

$$
\frac{\mathrm{d} Q_{1}}{\mathrm{~d} \phi}=\frac{1}{\omega} \alpha_{w} A_{w 1}\left(T_{w}-T_{1}\right)
$$

The output work made by the gas is defined as:

$$
\frac{\mathrm{d} W_{1}}{\mathrm{~d} \phi}=-P \frac{\mathrm{d} V_{1}}{\mathrm{~d} \phi},
$$

where the cylinder volume $V$ can be obtained from the standard slider-crank relationship.

The changing of mass in the control volume is satisfied the mass conservation deferential equation:

$$
\frac{\mathrm{d} m}{\mathrm{~d} \phi}=\frac{\mathrm{d} m_{i}}{\mathrm{~d} \phi}+\frac{\mathrm{d} m_{e}}{\mathrm{~d} \phi} .
$$

The instantaneous gas mass flow rate $\frac{\mathrm{d} m}{\mathrm{~d} t}$ can be calculated when the differential pressure and the open area of the valve port are determined. It is expressed as:

$$
\frac{\mathrm{d} m_{j}}{\mathrm{~d} t}=\mu A \sqrt{2 P_{\mathrm{I}} \rho_{\mathrm{I}}} \cdot \psi
$$

where subscript $j$ refers to the injection or exhaust gas property, replacing the subscripts $i$ and $e$ respectively. And the mass flow coefficient $\psi$ is determined by the conditions as follows:

When $\frac{P_{\amalg}}{P_{\mathrm{I}}}>\left(\frac{2}{\mathrm{k}+1}\right)^{\frac{\mathrm{k}}{\mathrm{k}-1}}$, it is in the sub-sonic flow state and the flow function is:

$$
\psi=\sqrt{\frac{\mathrm{k}}{\mathrm{k}-1}\left[\left(\frac{P_{\mathrm{U}}}{P_{\mathrm{I}}}\right)^{\frac{2}{\mathrm{k}}}-\left(\frac{P_{\mathrm{U}}}{P_{\mathrm{I}}}\right)^{\frac{\mathrm{k}+1}{\mathrm{k}}}\right]},
$$

When $\frac{P_{\amalg}}{P_{\mathrm{I}}} \leq\left(\frac{2}{\mathrm{k}+1}\right)^{\frac{\mathrm{k}}{\mathrm{k}-1}}$, it is in the sonic flow state and the flow function is:

$$
\psi=\left(\frac{2}{\mathrm{k}+1}\right)^{\frac{1}{\mathrm{k}-1}} \sqrt{\frac{\mathrm{k}}{\mathrm{k}+1}}
$$

Then, the mass flow rate differencing to the crank angle can be obtained by the equation:

$$
\frac{\mathrm{d} m_{j}}{\mathrm{~d} \phi}=\frac{1}{\omega} \frac{\mathrm{d} m_{j}}{\mathrm{~d} t}
$$

And the relations between the gas mass $\mathrm{m}$ and the state 
variables pressure $P$, temperature $T$ and volume $V$, are determined by the ideal gas equation of state:

$$
P V=m \mathrm{R} T
$$

Combining equations Eq. (7), Eq. (10) and Eq. (15), the thermodynamic sub-model of the first expansion stage is obtained.

\section{Sub-model of the second expansion stage}

Similarly, the process in the second control volume is analyzed. Here, the energy conservation differential equation can be obtained as:

$$
\begin{aligned}
\frac{\mathrm{d} T_{2}}{\mathrm{~d} \phi}= & \frac{1}{m C_{v}}\left[\frac{\mathrm{d} Q_{h}}{\mathrm{~d} \phi}+\frac{\mathrm{d} Q_{2}}{\mathrm{~d} \phi}+\frac{\mathrm{d} W_{2}}{\mathrm{~d} \phi}\right. \\
& \left.+h_{1} \frac{\mathrm{d} m_{e 1}}{\mathrm{~d} \phi}+h_{2} \frac{\mathrm{d} m_{e 2}}{\mathrm{~d} \phi}-u_{2} \frac{\mathrm{d} m_{2}}{\mathrm{~d} \phi}\right]
\end{aligned}
$$

The heat exchanges of the second control volume are calculated separately in the above equation, because the heat exchange coefficients are different in the heat exchanger and cylinder chamber. They are expressed as:

$$
\begin{aligned}
\frac{\mathrm{d} Q_{2}}{\mathrm{~d} \phi} & =\frac{1}{\omega} \alpha_{w} A_{w 2}\left(T_{w}-T_{2}\right) \\
\frac{\mathrm{d} Q_{e}}{\mathrm{~d} \phi} & =\frac{1}{\omega} \alpha_{w h} A_{w h}\left(T_{w}-T_{2}\right)
\end{aligned}
$$

The output work of the second cylinder is defined as:

$$
\frac{\mathrm{d} W_{2}}{\mathrm{~d} \phi}=-P_{2} \frac{\mathrm{d} V_{2}}{\mathrm{~d} \phi}
$$

The changing of mass in the control volume is also satisfied the mass conservation deferential equation:

$$
\frac{\mathrm{d} m_{2}}{\mathrm{~d} \phi}=\frac{\mathrm{d} m_{e 1}}{\mathrm{~d} \phi}+\frac{\mathrm{d} m_{e 2}}{\mathrm{~d} \phi}
$$

where the mass flow rate of the air injecting into and exhausting out of the second control volume also can be calculated by the equations Eq. (11) Eq. (14) separately. For the second control volume which is composed of the inner space of the heat recharge and the inner space of the second expansion stage cylinder, the gas equation of state of the air in the volume is given as:

$$
P_{2}\left(V_{\mathrm{h}}+V_{2}\right)=m_{2} \mathrm{R} T_{2}
$$

Combining equations Eq. (16), Eq. (20) and Eq. (21), the thermodynamic sub-model of the second expansion stage is obtained. As the equations show above, the two sub-models are joined by the air which expands in the two stage cylinders successively.

\section{Calculation of torque and efficiency}

The instantaneous torqueses of the two cylinders are expressed as follow equations:

$$
\begin{aligned}
M_{1}= & \frac{\left(P_{1}-P_{0}\right) S_{1}-m_{i 1} r \omega^{2}(\cos \phi+\lambda \cos 2 \phi)}{\cos \beta_{1}+\mu A_{b 1} \sin \beta_{1}} \cdot \sin \left(\phi+\beta_{1}\right) \cdot r \\
M_{2}= & \frac{\left(P_{2}-P_{0}\right) S_{2}-m_{i 2} r \omega^{2}(\cos (\phi+\pi)+\lambda \cos 2 \phi)}{\cos \beta_{2}+\mu A_{b 2} \sin \beta_{2}} \\
& \cdot \sin \left(\phi+\pi+\beta_{2}\right) \cdot r
\end{aligned}
$$

Now the total output torque of the engine $M_{t}$ goes as:

$$
M_{t}=M_{1}+M_{2}
$$

Then, the efficiency of the engine $\eta$ can be obtained by

$$
\eta=\frac{\int_{0}^{2 \pi}\left(M_{t} \cdot \omega\right) \mathrm{d} \phi}{\int_{0}^{2 \pi}\left(\frac{\mathrm{d} E_{i}}{\mathrm{~d} \phi}\right) \mathrm{d} \phi}
$$

Because the temperature of the injection air is assumed to be the same of $T_{0}$, the $E_{i}$ can be calculated as

$$
\frac{\mathrm{d} E_{i}}{\mathrm{~d} \phi}=\frac{\mathrm{d} m_{i}}{\mathrm{~d} \phi} R T_{0}\left(\ln \frac{P_{i}}{P_{0}}+\frac{P_{0}}{P_{i}}-1\right)
$$

\section{SIMULATION ANALYSIS OF BORE RATIO}

The step-fix 4-order Runge-Kutta method is used to calculate the differential equation sets of the model in the simulation program. The top dead center (TDC) of the first stage expansion cylinder is defined as the start point of the cycle and the simulation step is $0.01^{\circ}$. The main parameters of the engine are defined in table 1 .

Table 1 Parameters settings of the APE

\begin{tabular}{|l|l|}
\hline \multicolumn{1}{|c|}{ Parameters } & \multicolumn{1}{c|}{ Value } \\
\hline Bore of the $1^{\text {st }}$ stage cylinder & $50 \mathrm{~mm}$ \\
\hline Piston stroke & $49.5 \mathrm{~mm}$ \\
\hline Length of connecting rod & $170 \mathrm{~mm}$ \\
\hline Ambient temperature & $20^{\circ} \mathrm{C}$ \\
\hline Ambient pressure & $0.101 \mathrm{MPa}$ \\
\hline Injection air pressure & $1.2 \mathrm{MPa}$ \\
\hline Inside diameter of the heat-exchanger & $20 \mathrm{~mm}$ \\
\hline Length of the heat-exchanger & $200 \mathrm{~mm}$ \\
\hline Speed of the APE & $1000 \mathrm{r} / \mathrm{min}$ \\
\hline
\end{tabular}




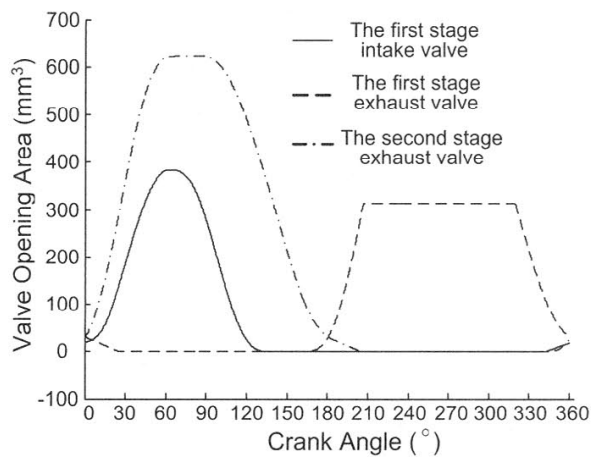

Figure 2 Valve opening area curves

And the curves of valve opening area in an operating cycle are defined by the port timing as Figure 2 shows. In those curves, the maximum opening area of the second stage exhaust valve is limited by the sectional area of the heat-exchanger.

The bore of the first stage cylinder is fixed while the bore of the second stage cylinder is assigned different values to define different bore ratios. Now the bore ratios are changing from 1.1 to 1.6 with the step of 0.1 in the simulation.

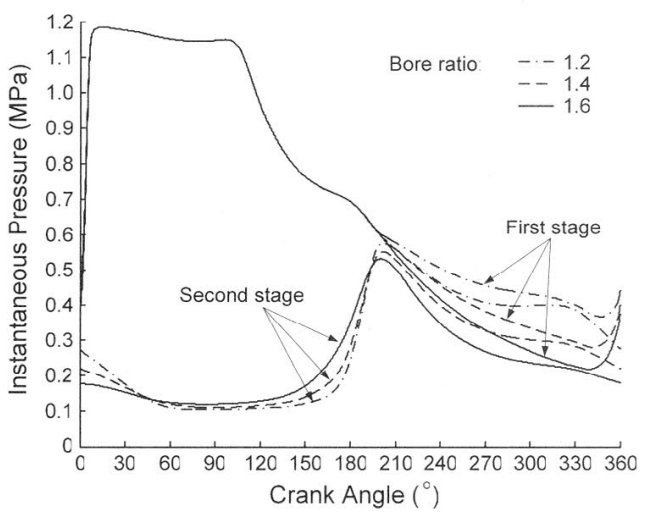

(a) Instantaneous in-cylinder pressures

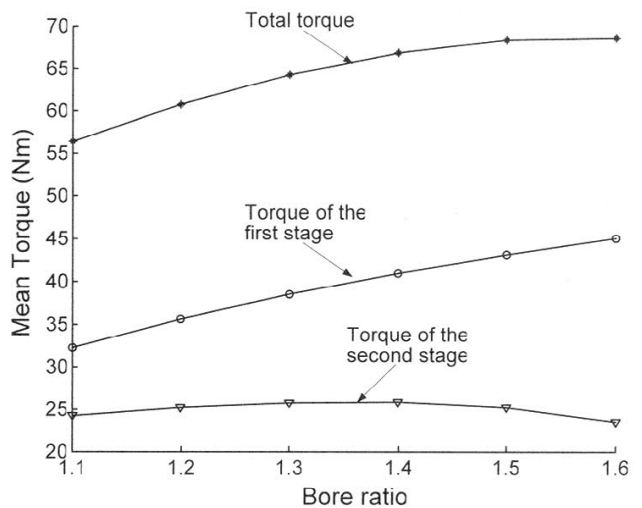

(b) Mean Torque

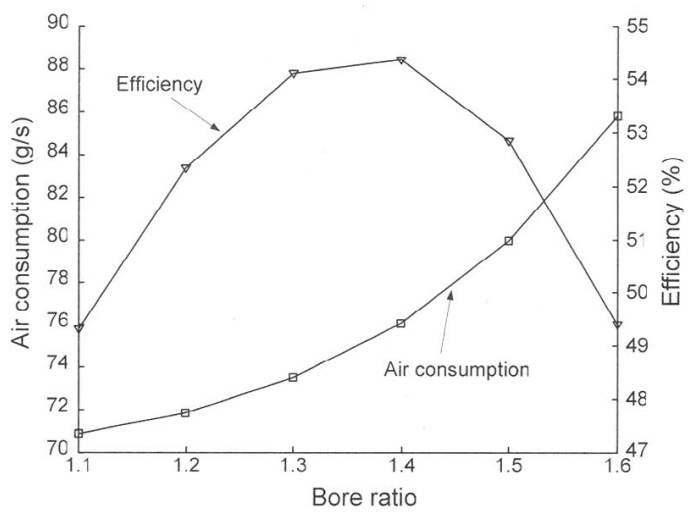

(c) Air consumption and efficiency

Figure 3 Simulation results under different bore ratio

The results of simulation come as Figure 3, which show the effect of bore ratio on the performance of TSEAPE. The total torque of the engine appears increasing with the rising bore ratio while its increase rate is dropping. And the efficiency of the engine increases with the rising bore ratio at the beginning but drops too when the bore ratio becomes too big. As figure 3(c) shows, the efficiency will reach the peak near the point that bore ratio is 1.4 , where output torque of the engine can express a nice too

\section{EXPERIMENTS OF TSEAPE}

With the indication of the simulation results, experiments were carried out to study the features of the TSEAPE. A production SI engine, which was designed to operate with gasoline, was modified so as to work with high-pressure air in the two-stage expansion mode. After adding lining and reboring, the bore sizes of the four cylinders were changed from originally all of $92 \mathrm{~mm}$ to two of $72 \mathrm{~mm}$ and two of $98 \mathrm{~mm}$. Therefore, the bore ratio of this prototype TSEAPE is 1.36 that comes near the best value appearing in the simulation results.

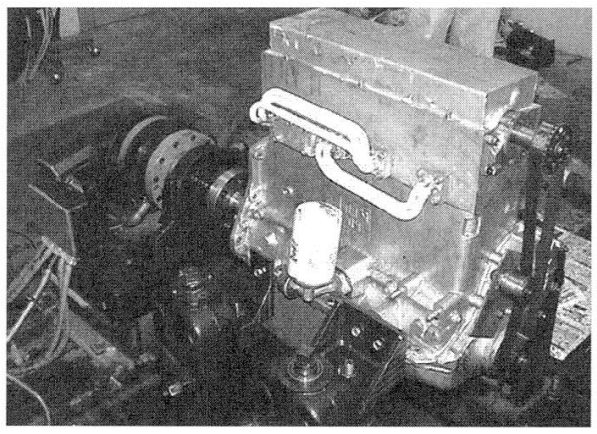

Figure 4 TSEAPE on the test bench 
The cylinder end of the engine that contains air distribution system is completely redesigned. From the left end, the first cylinder is cascaded with the third cylinder and the second cylinder is cascaded with the fourth cylinder by heat-exchanges. The engine is installed in a test bench for typical dynamometer experiments, as Figure 4 shows.

Experiments were carried out and the results are exhibited in Figure 5. The prototype TSEAPE under this bores ratio does run stably on air and it appears better power output and efficiency under relatively higher loads. But it is hard to say that its performance is good enough. The main reason is that the prototype TSEAPE is converted from an old combustion engine whose body does not fit the air powered operation mode very much, as carefully analysis after the experiments. The big inertia force and friction force caused by heavy crankshaft and tight bearing waste lots of the power. Those high-strength structures are unnecessary because the pressure in the cylinders is much lower than combustion engine and also the temperature.

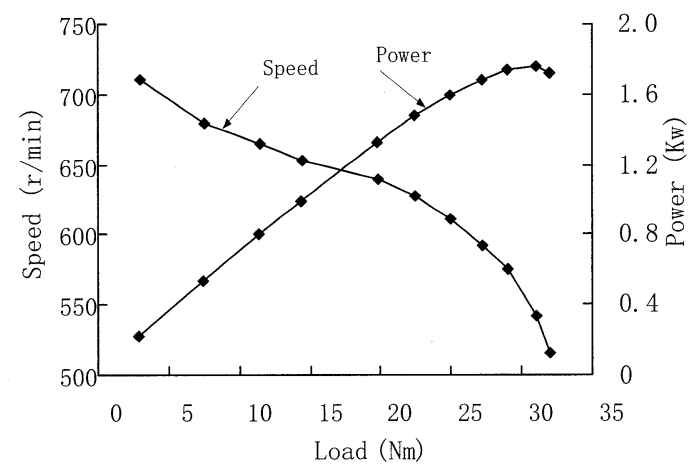

(a) Speed and power

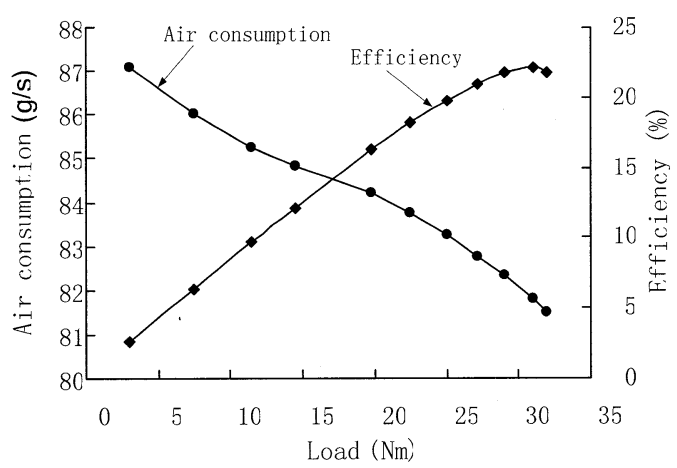

(b) Air consumption and efficiency

Figure 5 Experiment results under different loads

On the other hand, the surface of the heat exchange becomes heavily frosting soon after the engine working, which can be seen in the Figure 5. It shows that the air exhausted from the first stage cylinders is very cold and does have drastically heat exchange with the ambient when it pass the copper pipes. However, frost that covers the pipes will reduce their heat exchange ability.

Considering the reasons above, the experiment results is not far from the simulation and the analysis based on the model can be approximately approved.

\section{CONCLUSIONS}

Bores ratio represents the ratio of expansion volumes of cylinders that cascaded in a TSEAPE and it is a key structure parameter. Based on the model of TSEAPE that has been developed on the thermodynamics and mechanics analysis, the effects of bores ratio on the performance of TSEAPE are investigated by simulation in this paper. The simulation results indicate that the TSEAPE will have a good performance when its bore ratio is about 1.4, which is a good balance between the power output and the efficiency. The experiments carrying out on a prototype TSEAPE with bore ratio 1.4 show that it runs stably on air and appears better power output and efficiency under relatively higher loads. But probably due to the large inertia force and friction force caused by the high-strength structures of the combustion engine body, the energy wasting of the prototype TSEAPE is huge. And thick frost appears and covers the heat exchangers that reduce their heat exchange ability. So the performance of the engine is far from perfect. Considering those disadvantage above, the experiment results go near to the simulation analysis.

It is very important to choose appropriate bores ratio in TSEAPE designing. The method and the result obtained in this paper are of good help for that. And the problems appear in the experiments are some of the key research points that will be solved in the near future.

\section{REFERENCES}

1. Chen, Y., Xu, H., Tao, G. L., Wang, X. Y., Liu, H. and Jia, G. Z., Research and progress of the compressed air powered vehicle, Chinese Journal of Mechanical Engineering, 2002, 38-11, pp.7-11 (in Chinese).

2. Liu, L. and Yu, X. 1., Optimal design of ideal cycle in air powered engine, Journal of Zhejiang University ( Engineering Science), 2006 ,40-10, pp.1815-1818.

3. Ordonez C A, Plummer M C, Reidy R F., Cryogenic heat engines for powering zero emission vehicles. Proceedings of 2001 ASME International Mechanical Engineering Congress and Exposition, New York: [ASME], 2001.

4. Knowlen, C., Mattick, A. T. and Bruckner, A. P., High efficiency energy conversion systems for liquid nitrogen automobiles, SAE Paper, 1998, No. 981898.

5. Website of MDI Company, http://www.theaircar.com , 2008 